\title{
Primary mucinous cystadenocarcinoma of renal pelvis: a case
} report

\author{
Mehdi Fareghi ${ }^{1}$, Afshin Mohammadi*2 and Kazem Madaen ${ }^{1}$
}

\begin{abstract}
Address: ${ }^{1}$ Urology Department, Imam Reza University Hospital, Tabriz University of Medical Sciences, Tabriz, Iran and ${ }^{2}$ Radiology Department, Imam Khomeini University Hospital, Urmia University of Medical Sciences, Urmia, Iran

Email: Mehdi Fareghi - mfareghi@yahoo.com; Afshin Mohammadi* - mohamadi_afshin@yahoo.com; Kazem Madaen - kmadaen@yahoo.com

* Corresponding author
\end{abstract}

Published: 23 December 2009

Cases Journal 2009, 2:9395 doi:10.1 186/1757-1626-2-9395

This article is available from: http://www.casesjournal.com/content/2/1/9395

(C) 2009 Fareghi et al; licensee BioMed Central Ltd.

This is an Open Access article distributed under the terms of the Creative Commons Attribution License (http://creativecommons.org/licenses/by/2.0), which permits unrestricted use, distribution, and reproduction in any medium, provided the original work is properly cited.

\begin{abstract}
Background: We report a case of primary mucinous cystadenocarcinoma of renal pelvis which radiologically resembled large multicystic mass in 45 years old man.

Case Presentation: The patient referred to our center with loin pain and progressive abdominal distention from 4 years ago. In the previous published literature, four cases of mucinous cystadenocarcinoma of renal origin have been published. Abdominal CT showed complete replacement of left kidney by a large multiloculated cystic mass accompanied with multiple large nephrolithiasis. Nephrectomy was performed and histopathology revealed covering of epithelium of renal pelvis by columnar epithelium and scattered goblet cells and mucous gland.

Conclusion: Mucinous cystic neoplasms of kidney are rare entity and our case and few similar reported cases showed that this tumor is an unique clinicopathologic renal mass that may be classified by World Health Organization classification in the future
\end{abstract}

\section{Background}

The most common neoplasms in the renal pelvis are urothelial in origin, although infrequently, squamous and glandular neoplasm may arise within the renal pelvicalyceal system through metaplastic transformation of the urothelium due to long-standing obstruction and infection and chronic irritation by urolithiasis [1]. In previous published literature invasive mucinous adenocarcinoma has been well documented [2,3], but malignant mucinous cystic tumors are exceedingly rare primary neoplasms involving renal pelvicalyceal system with only 4 cases reported in the literature [4-7].

Because knowledge about renal mucinous cystic neoplasms is extremely limited and they have not been recognized in the World Health Organization classification of pelvicalyceal system malignancy. We describe the clinical, radiological and pathological findings of a 45 years old man with mucinous cystadenocarcinoma arising in a background of extensive mucinous metaplasia of pelvicalyceal urothelium secondary to probably chronic nephrolithiasis and hydronephrosis.

\section{Case Presentation}

A 45-year-old man presented with a history of a dull aching pain in the left flank and progressive abdominal distention since 4 years ago. Recently the patients complain from aggravation of abdominal pain and new onset constipation. He had no history of passing stones. He had not history of haematuria, dysuria, frequency or fever during 4 years ago. 
General physical examination revealed nothing positive. Abdominal examination revealed asymmetrical buldging of abdomen on inspection and a firm fixed mass in the left hypochondrium that extended to the epigastrium and hypochonderium on palpation. (Figure 1)

Laboratory investigations showed haematuria, mucosuria and 10-12 pus cells/high power filed in urine examination. Urine culture was negative.

Routine hematological investigations were within normal limit.

Intravenous pyelography revealed the non-functioning left kidney with multiple large opaque stones. In transabdominal sonography a huge cystic mass with internal echo debris and multiple stones without remarkable renal parenchyma was observed that mimicked sever longstanding hydronephrosis accompanied with pyonephrosis.

Abdominal CT scan (64-MDCT, Siemens. Somatom, Sensation) with IV and Oral contrast revealed a huge lobulated multicystic thin wall mass measuring $25 * 20 * 30 \mathrm{cms}$ with thick septation accompanied with solid component without marked enhancement and some large nodular calcified density (stone) that completely replaced left kidney. (figure 2).

Abdominal CT did not reveal any other mass lesions or ascites in the abdomen. There was no singe of hydroureter in abdominal sonography and abdominal CT scan.

Surgical excision of the mass was performed completely. Grossly left kidney was completely replaced by lobulated

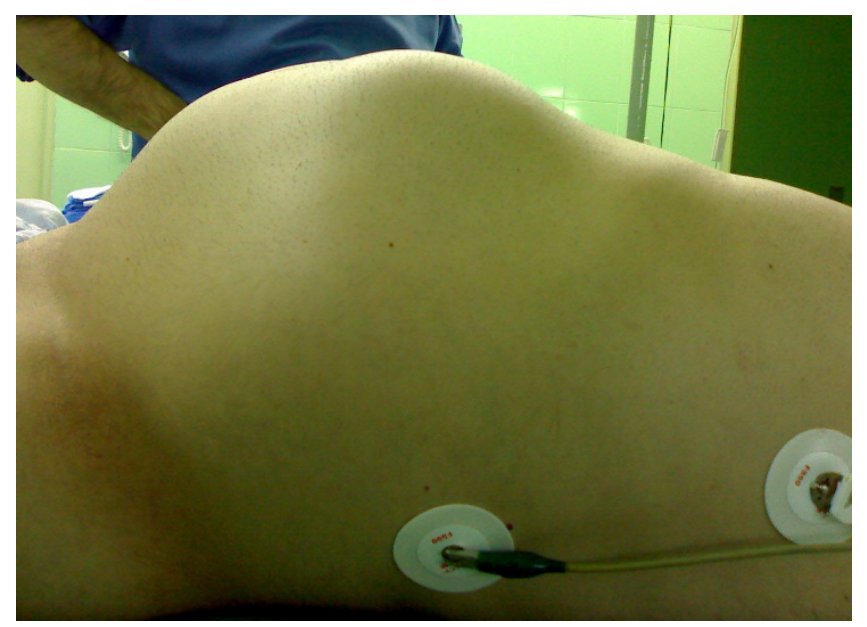

Figure I

Image show severe abdominal distention of a 45 year's old man.

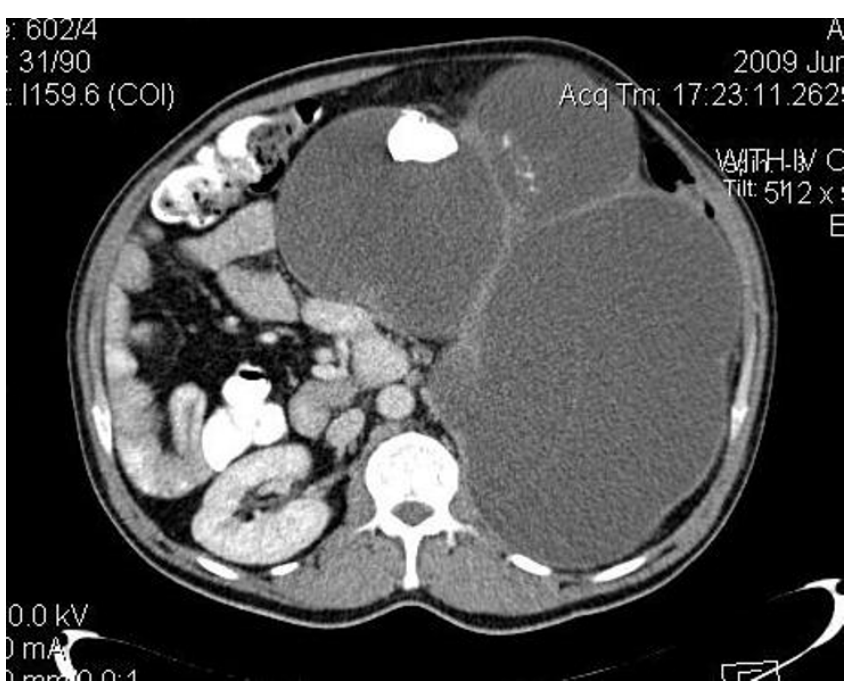

Figure 2

Axial image show huge multiloculated cystic mass with a large nephrolithiasis and thick septation.

multicystic mass measuring $30 * 20 * 20 \mathrm{cms}$ that were filled with necrotic and mucinous material. (Figure 3 ).

Histopathology revealed that the tumor was composed of single layer of columnar epithelium with scattered goblet cells and mucous glands resemblance to intestinal mucosa with abundant extracellular mucin compatible with cystic mucinous adenocarcinoma (Figure 4).

The renal capsule, Ureter and renal hilar vessels were intact (T 3).

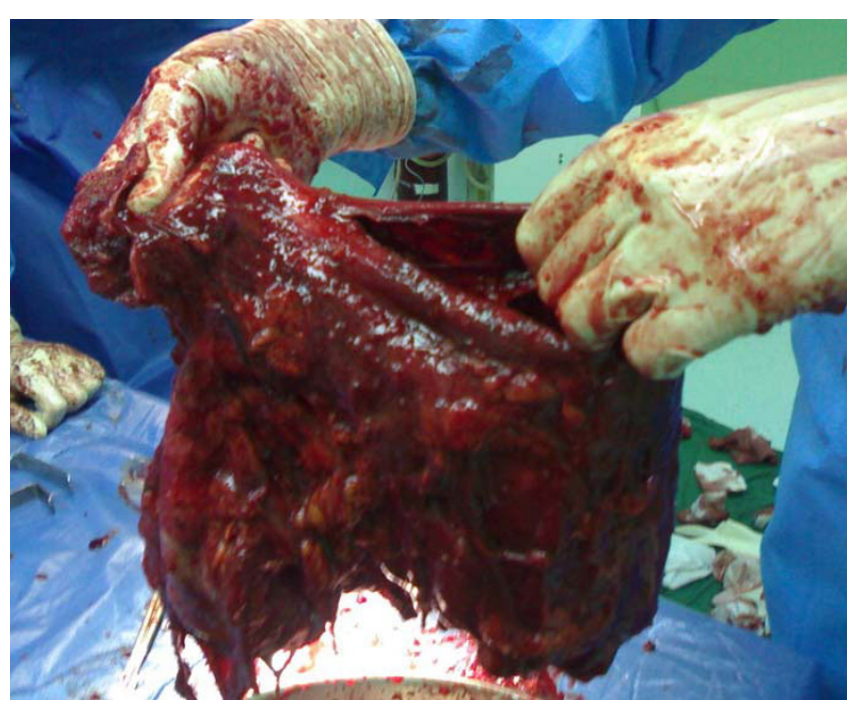

Figure 3

Photograph show gross appearance of a complex multiloculated cystic mass replacing the entire left kidney. 


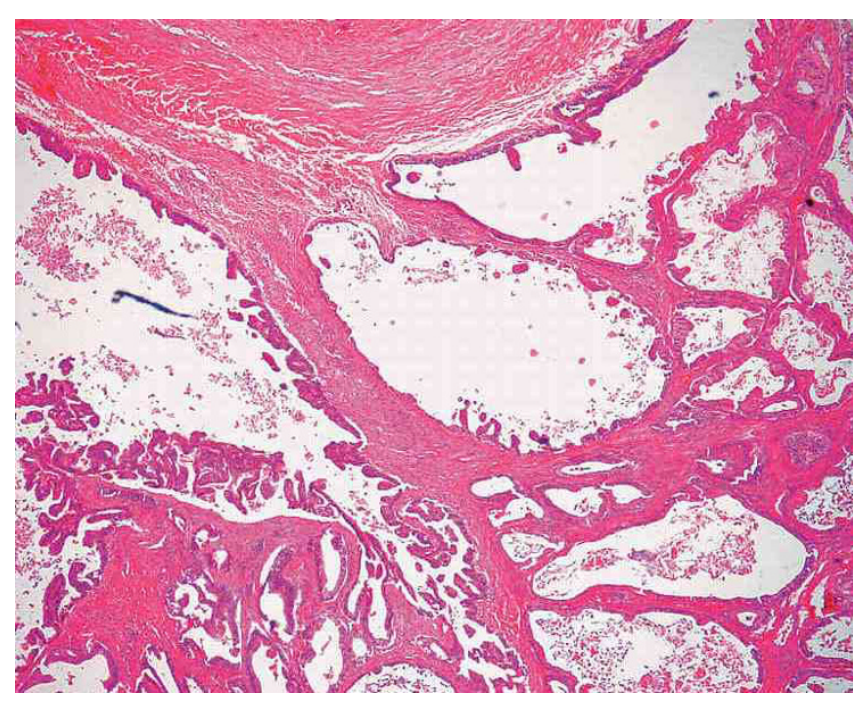

Figure 4

Cystadenocarcinoma showing variously sized cystic structures lined by atypical, but well-differentiated mucin-producing glandular epithelium, focally forming papillary structures.

\section{Discussion}

Tumors of the renal pelvis are uncommon, with relative frequency of transitional cell carcinoma (90\%), squamous cell carcinoma (10\%) and adenocarcinoma (1\%) [1]. Mucinous cystic neoplasms of kidney are exceedingly rare primary neoplasm, with only 5 benign, 3 borderline [7] and 4 malignant [4-7] cases reported in the literature. Mucinous cystadenocarcinima of renal pelvis was first described in 1960 by Hasebe et al.

These tumors occur as a result of metaplasia of the transitional epithelium of the calyces and pelvis into glandular epithelium, which then undergoes a malignant transformation [8]. Presence of chronic infection, hydronephrosis and calculi are commonly associated with these tumors [9]. Our patient had multiple large stone predisposing him to all the above conditions. Some authors postulated that formation of the calculi might be the result of over secretion of glycoproteins by the tumor and binding of that with cations such as sodium, calcium, and magnesium, forming larger calculi. Thus, calculi may not be the cause of the neoplasm [10].

Patients are often asymptomatic. Hematuria is the most common presenting sign and loin pain and palpable abdominal mass are the late presentation of this tumor[7]. Our patient had no history of gross or microscopic hematuria.

Radiological studies may not be able to identify malignant tumor [1]. In our patients neither ultrasonography nor abdominal CT scan were not suggestive for malignancy. According to imaging finding long-standing UPJO and chronic hydronephrosis due to nephrolithiasis was the most probable diagnosis of our patient. Because mucinous cyst adenocarcinoma is an exceedingly rare entity a careful search for a primary carcinoma originating elsewhere such as pancreas, ovary and appendix should be excluded. In our patient the patient was male and radiologic evaluation of pancrease and appendix was negative for malignancy.

Mucinous cystic neoplasms of kidney are rare entity and our case and few similar reported cases shwe that this tumor is an unique clinicipathologic renal mass that may be classified by World Health Organization classification in the future.

\section{Consent}

Written informed consent was obtained from the parents of the patient for publication of this case report and accompanying images. A copy of the written consent is available for review by the Editor-in-Chief of this journal.

\section{Competing interests}

The authors declare that they have no competing interests.

\section{Authors' contributions}

MF and KM were the attending doctor, carried out the surgical procedure and literature review. AM was the attending doctor and collected the data and literature review, and wrote the manuscript. All authors read and approved the final manuscript.

\section{References}

I. Kaur G, Naik VR, Rahman MNG: Mucinous adenocarcinoma of the renal pelvis associated with lithiasis and chronic gout. Singapore Med J 2004, 45(3): I25-126.

2. Spires SE, Banks ER, Cibull ML, Munch L, Delworth M, Alexander NJ: Adeocarcinoma of renal pelvis. Arch Pathol Lab Med 1993, I 17: I I56-60.

3. Park S, Meng MV, Greenberg MS, Deng DY, Stolle ML: Muconephrosis. Urology 2002, 60(2):344.

4. Hasebe M, Serizawa S, Chino S: One a case of papillary cystadenocarcinoma following malignant degeneration of a papillary adenoma in the kidney pelvis. Yokohama Med Bull 1960, II:49I-500.

5. Mardi K, Sharma J, Mahajan P: Mucinous cystadenoma of renal pelvis with malignant transformation: a case report. Indian J Pathol Microbiol 2006, 49:595-596.

6. Muraoka K, Osada Y: Cystadenocarcinoma of renal origin in a supernimery kidney. Ryoikibetsu Shokogun Shiizu 1997:453-455.

7. Rao P, Pinheiro N Jr, Franco M, Ra S, Costa H, Manzano , Paner GP, Silva EG, Amin MB: Pseudomyxoma peritonei associated with primary mucinous borderline tumor of the renal pelvicalyceal system. Arch Pathol Lab Med 2009, 133(9):1472-6.

8. Kakkar N, Joshi K, Mandal AK: Mucinous adenocarcinoma of the renal pelvis: A case report. Indian J Pathol Microbiol 1997, 40:393-5.

9. Shah V B, Amonkar G P, Deshpande J R, Bhalekar H: Mucinous adenocarcinoma of the renal pelvis with pseudomyxoma peritonei. Indian J Pathol Microbiol 2008, 5 I (4):536-537.

10. Liwnicz BH, Lepow H, Schutte H, Fernandez R, Caberwal D: Mucinous adenocarcinoma of the renalpelvis: discussion of possible pathogenesis. J Urol 1975, I 14:306-10. 